\title{
Application of microwaves in the convective drying of ceramic
}

\section{Rajewska, K. ; Pawłowski, A.}

Institute of Technology and Chemical Engineering, Poznań University of Technology, ul. Berdychowo 4, 60-965 Poznań, Poland

*E-mail of the corresponding author: kinga.rajewska@put.poznan.pl

\begin{abstract}
The paper demonstrates microwave and convective drying processes in different combinations of kaolin cylindrical samples in laboratory scale. Analysis of the kinetics and sample temperature evolution show that the moment of application and electromagnetic field duration have the biggest influence on characteristics of the drying curve and quality of dried material. Dried samples were subjected to strength tests using the Brazilian method in order to determine the relation between its quality and different drying programs.
\end{abstract}

Keywords: ceramic, convective drying, microwave drying, strength test. 


\section{Introduction}

The use of microwave technology in industrial chemical processing has been developed since the 1970s. This is related to the general tendency to reduce production costs and care for the environment. One of the industrial processes using microwaves as a source of heat is drying. Microwave energy, alone or in combination with conventional energy sources (hybrid methods) enables precise control of the drying process in order to achieve higher efficiency and better product quality in the shortest possible time. In conventional drying, heat is transported to the surface of the material by conduction, convection or radiation and into the material through heat conduction. Moisture is initially evaporated from the surface, and the remaining water diffuses to the surface. This is often a slow process and the diffusion rate is limited. Process time is determined by the heat flow rate in the material from the surface, which depends on its specific heat, thermal conductivity, density and viscosity. Microwaves as a form of thermal energy, manifest themselves through their interaction with materials. The electromagnetic field initially interacts with the outer layers of the material. The inner part of the material is heated when the heat moves from the outer layers to the inside. Most of the moisture evaporates before leaving the material. If the material is very wet and the pressure inside is rapidly increasing, the fluid will be removed from the material due to the pressure difference. This creates a kind of pumping action that ejects the liquid to the surface, often in the form of steam. This results in very fast drying without the need to overheat the atmosphere and emit greenhouse gases from the heat source. The energy is transferred by the material to the electromagnetic way, not as a heat flux. Therefore, the heating rate is not limited, and the uniformity of heat distribution is significantly improved.

In general terms, according to literature data, the group of materials processed using microwave energy includes: food, plants, textiles, wood, soils and other biological materials [n1]. Among the works concerning the use of microwaves in drying, one can find a group of publications regarding the use of dielectric heating in industrial processes [n2, n3, n4]. Another group of publications are purely experimental works concerning both kinetics and material quality $[\mathrm{n} 5, \mathrm{n} 6]$. The next set of works are articles binding theoretical modeling of the drying process with experiments [n7, n8, n9, n10]. In the works that take into account the issue of the quality of the dried material, the emphasis is mainly on the effect of reducing the mechanical properties. The papers [n11, n3, n5] mainly concern the theoretical analysis of volumetric, intermittent drying, which is to reduce the gradients of moisture distribution, and hence the smaller deformations of the dried products. The theoretical part concerns analysis of stresses generated during drying. In turn theoretical work [n12] contains a very detailed and advanced model of the dielectric drying process. 
The presented work concerns the investigation of the influence of microwaves on drying kinetics and mechanical strength of kaolin after drying, as the estimation of the quality of microwave dried products only by surface examination may not be sufficient

\section{Materials and Methods}

The material used in the research was ceramic kaolin. A slurry was prepared with a moisture content of $0.4 \mathrm{~kg} / \mathrm{kg}_{\mathrm{db}}$. Then a sample was formed with cylindrical geometry (diameter - $60 \mathrm{~mm}$, height - $60 \mathrm{~mm}$ ). In the dryer it is possible to control the speed and temperature of the air entering the chamber and the microwave power. Temperature measurements were made using optical fibers in two places: in the axis, half the height of the cylinder $\left(\mathrm{T}_{\mathrm{in}}\right)$ and at a distance of $0.5 \mathrm{~cm}$ from the upper surface of the cylinder $\left(\mathrm{T}_{\text {out }}\right)$.The strategy of combining the above-mentioned drying methods used in this work was partly based on Schiffmann's proposal [n13].

As part of the work, seven different drying programs were carried out. The speed and temperature of the air entering the drying chamber were changed, as well as the stage in which the microwave energy process was supported (Table 1). The criterion of the end of the process was to obtain a sample moisture of $0.06 \mathrm{~kg} / \mathrm{kg}_{\mathrm{db}}$. Regardless of the drying strategy, the critical moisture content corresponding to the theoretical boundary between the constant and decreasing drying rate, was on average $0.23 \mathrm{~kg} / \mathrm{kg}_{\mathrm{db}}$. Additionally, the mechanical strength of the samples was analyzed using the Brazilian method.

Table 1. Process data

\begin{tabular}{lccl}
\hline & $\mathbf{v}$ [m/s] & $\mathbf{T}\left[{ }^{\circ} \mathbf{C}\right]$ & application of $\mathbf{M W}-\mathbf{1 0 0} \mathbf{~ W}$ \\
\hline Exp.1 & 3 & 80 & - \\
Exp.2 & 3 & 80 & the whole process \\
Exp.3 & 3 & 80 & constant rate period \\
Exp.4 & 3 & 80 & falling rate period \\
Exp.5 & 3 & 26 & the whole process \\
Exp.6 & 3 & 26 & constant rate period \\
Exp.7 & 2 & 26 & the whole process \\
\hline
\end{tabular}

\subsection{Results and discussion}

As a graphical presentation of the process, the drying curves, the drying rate and the temperature of the material were presented, because these parameters show sufficiently the kinetics of the process. The first was to perform convection drying without microwave energy support (Fig. 1). 

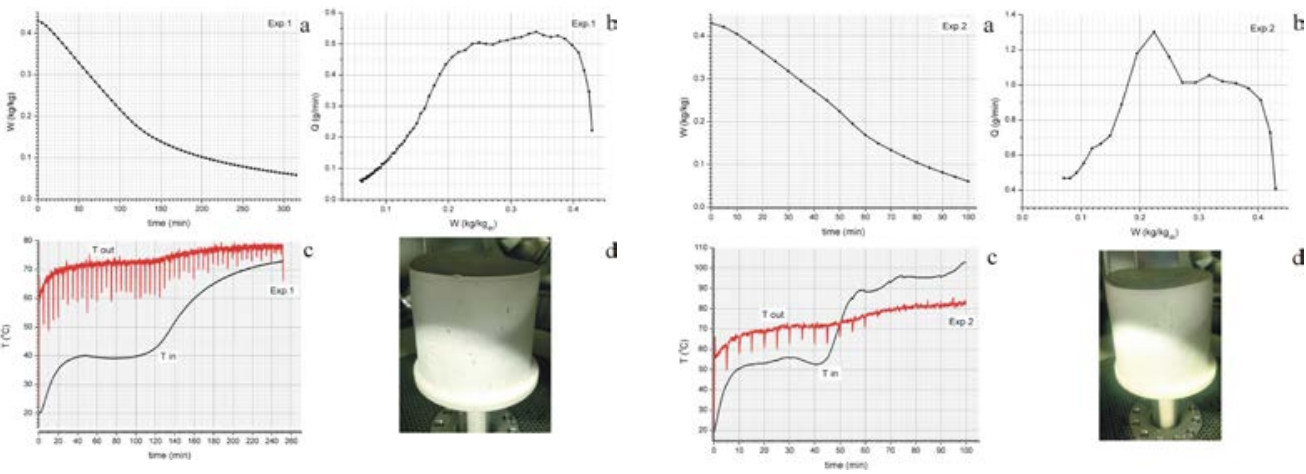

Fig. 1 Experiment 1.

Fig. 2 Experiment 2.
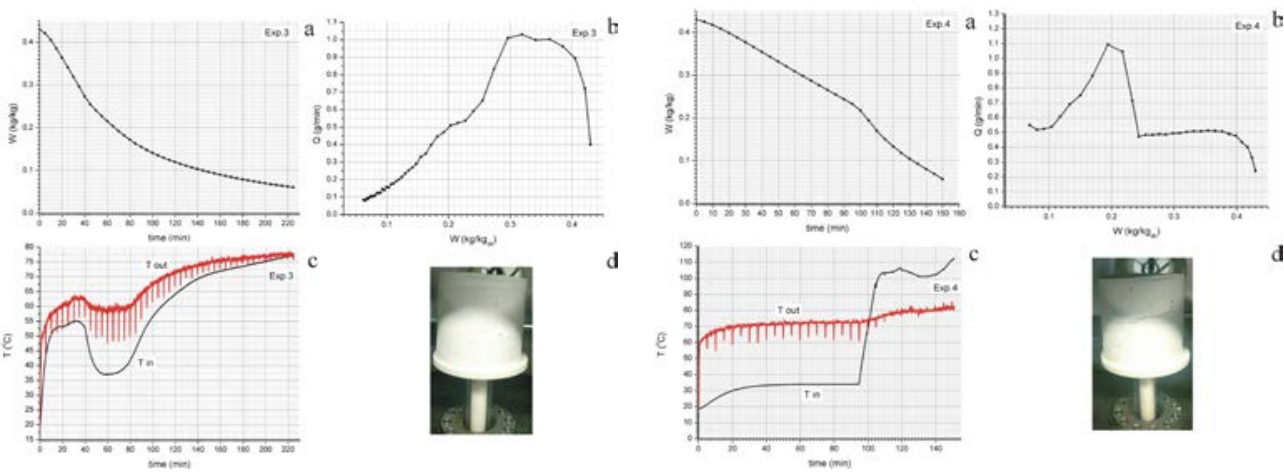

Fig. 3 Experiment 3.

Fig. 4 Experiment 4.
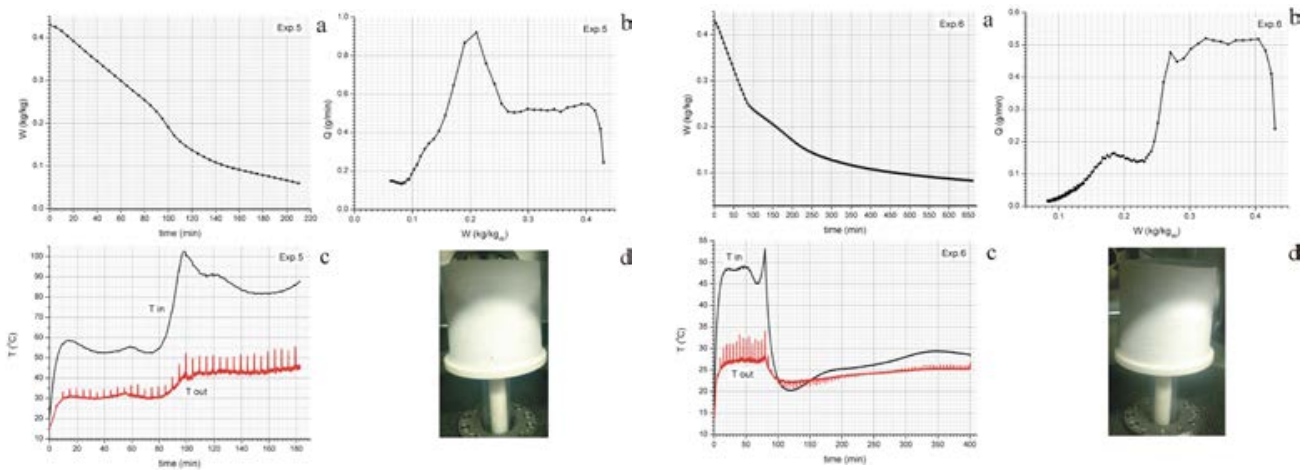

Fig. 5 Experiment 5.
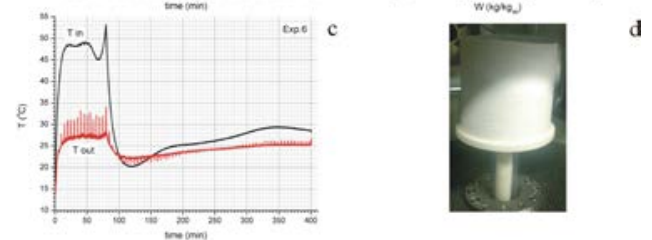

Fig. 6 Experiment 6. 
The heating period is clearly visible, a period of constant drying rate at which the material temperature and the drying rate are constant and amount to $0.5 \mathrm{~g} / \mathrm{min}$. Then the process enters the second phase, the drying rate decreases and the temperature of the material increases. The final drying criterion is reached after five hours, the material temperature reaches $73^{\circ} \mathrm{C}$. In the sample photograph taken after the end of the process (Fig. 1d), a surface crack running circumferentially about $0.5 \mathrm{~cm}$ under the edge of the upper surface of the cylinder can be seen. Fig. 2 illustrates the course of the process with continuous exposure of the material to the microwaves. The total drying time is three times shorter and the drying speed in the first phase is twice as high as in Exp. 1. Also, the temperature of the material being subjected to microwave treatment is higher and amounts to $52^{\circ} \mathrm{C}$ during a constant drying rate. At the end of this phase, the drying rate increases rapidly, and after reaching critical moisture content, it decreases to the end of the process. The material temperature reaches $103^{\circ} \mathrm{C}$. Small circumferential cracks can be seen on the side of the sample. A further drying strategy is shown in Fig. 3. Microwave energy enhances convective drying during heating and constant drying rates. After turning off the microwave generator, the drying rate and material temperature decreases accordingly, however, because the average moisture content in the material has not yet reached the critical value, the phase of the constant drying rate is continued, but under conditions with Exp. 1. At the end of the process, the material temperature reaches about $77^{\circ} \mathrm{C}$. In the upper part of the cylinder, a distinctive characteristic circumferential crack was again created without a net of smaller cracks.

In Experiment 4, microwaves were used during the decreasing drying rate of the period (Fig.4). Initially, the drying rate was $0.5 \mathrm{~g} / \mathrm{min}$ (according to data from Exp.1), after turning on the microwave generator the drying rate increased sharply with the increase of the material temperature. Then, with the decrease of the moisture content, the drying rate decreased and the material temperature reached $120{ }^{\circ} \mathrm{C}$ at the end of the process. The dried sample according to this strategy has been destroyed. In addition to the crack located circumferentially in the upper part, a deep circumferential fracture in the middle of the heights dividing the sample into two parts appeared.

The next three processes were carried out with the heater turned off. In Exp. 5 (Fig.5), the

energy of the electromagnetic field enhances the entire process. The total drying time increased in relation to a similar process, but with the heater turned on (Exp.2). The material temperature during the constant drying rate was $55{ }^{\circ} \mathrm{C}$. As in the case of Experiment 2, the mass flux doubled in the final stage of the constant drying rate and then the temperature of the material reached a maximum value of $102{ }^{\circ} \mathrm{C}$. The dried sample was free of any defects visible to the naked eye.

In Experiment 6 (Fig.6), the microwaves were turned on during the heating period and the 
constant drying rate. The drying rate was maintained at $0.5 \mathrm{~g} / \mathrm{min}$. After switching off the microwaves, it decreased to $0.15 \mathrm{~g} / \mathrm{min}$ and was maintained until critical humidity was reached and then decreased. The temperature of the material was maintained at about $50{ }^{\circ} \mathrm{C}$ in the first drying period, then it dropped after switching off the microwaves to the temperature of the drying medium, i.e. around $25^{\circ} \mathrm{C}$. The sample, similarly to the previous process, has no scratches or cracks potentially weakening the material.

In the last process the air velocity was reduced from $3 \mathrm{~m} / \mathrm{s}$ to $2 \mathrm{~m} / \mathrm{s}$. Reducing the speed of the drying medium caused the temperature of the wet thermometer to increase by $5{ }^{\circ} \mathrm{C}$ compared to a similar process with higher airflow (Exp. 5). However, the remaining process parameters such as the total drying time and the mass flux rate remained unchanged. A few small scratches are visible on the surface of the dried sample.

Analyzing the temperature curves of the dried sample (Fig. 1c-6c), some characteristic steps can be observed. They illustrate how to use the energy delivered to the material by convection, with microwaves or in combination. During the heating period, the temperature of the material and water contained in it increases (sensible heat). At the same time, in the final phase of the heating period volatile substances start to evaporate (latent heat). The start of the evaporation process therefore slows down the temperature increase. Next comes the equilibrium stage - constant temperature of the material. Latent heat of evaporation absorbs most of the energy. The process is in the constant phase of the drying rate. The next stage of the temperature rise occurs due to evaporation of most volatile components. Sensible heat absorbs most of the energy supplied. A phase of decreasing drying rate follows. The drying process from the point of view of mass transport is best illustrated by the drying rate curves (Fig. 1b-6b). In strategies with continuous microwave assist (Exp. 2, 5,7 ), a sudden increase in mass flux was noted at the final stage of the constant drying rate period when the evaporation surface moves back into the material. The temperature and pressure in the pores increase, which results in a temporary acceleration of the rate of moisture removal from the deeper layers of the material. This effect is not observed in a conventional process. After a sudden increase, the temperature stabilizes and the process enters the final stage of decreasing speed. Then the water remains are removed from the deeper layers of the material. The moisture diffusion path in the pores lengthens and the capillary processes play a greater role in mass transport.

In the visual assessment of the dried samples, a characteristic circumferential crack is always observed near the upper surface of the cylinder in the case of drying with hot air. This is the result of the greatest drying stresses occurring there. The destructive power determined in the Brazilian test is also lower in these processes: $350 \mathrm{~N}$ (Exp.1), $280 \mathrm{~N}$ (Exp. 2), $320 \mathrm{~N}$ (Exp. 3), than in processes without convection heating: $530 \mathrm{~N}$ (Exp 5) and $450 \mathrm{~N}(\operatorname{Exp} 7)$. 


\section{Conclusions}

The use of microwaves during drying results in undoubtedly shortening the drying time, but also an increase in temperature, which is not always beneficial. The application of microwave energy in the phase of decreasing drying rate (one of Schiffmann's proposals) does not work in the case of brittle materials with the geometry used in this work.

The drying strategies used in this work have a significant impact on both the drying time and the final quality of the product.It turns out that the best strategy due to the drying time and the quality of the material is the use of microwave support throughout the process, but without convection heating. The drying time has been shortened and the quality improved compared to conventional drying. These tests do not provide enough information when and how long to turn on microwaves to achieve optimal results. They only indicate that it is possible to shorten the process time and even improve the quality of the material.

\section{Nomenclature}

$\begin{array}{lll}\mathrm{T} & \text { temperature } & { }^{\circ} \mathrm{C} \\ \mathrm{W} & \text { moisture content } & \mathrm{kgkgdb}^{-1} \\ \mathrm{Q} & \text { drying rate } & \mathrm{gmin}^{-1}\end{array}$

Subscripts

$\begin{array}{ll}\text { in } & \text { inside } \\ \text { out } & \text { outside } \\ \mathrm{db} & \text { dry body }\end{array}$

The paper was developed under project no. 03/32/DSPB/0805 financed by the Poznan University of Technology.

\section{References}

[1] Mujumdar, A.S. ed., Handbook of industrial drying, fourth edition, Taylor \& Francis Group, 2015.

[2] Tinga, W.R.; Nelson, S.O. Dielectric properties of materials for microwave processing - tabulated, Journal of Microwave Power 1973, 8(1), 23-65.

[3] Schiffmann, R.F. Microwave and Dielectric Drying in A.S. Mujumdar (Ed.): Handbook of Industrial Drying, Marcel Dekker, New York, USA, 1987.

[4] Metaxas, A.C. Microwave heating, Power Engineering Journal, 1991.

[5] Itaya, Y.; Uchiyama, S.; Hatano, S.; Mori, S. Drying enhancement of clay slab by microwave heating. In proceedings of IDS, Brazylia, 2004. 
[6] Lei, L.Y.; Zhang, Y.L.; Peng, J.; Li, C. Microwave dryling characteristics and kinetics of ilmenite. Transactions Nonferrous Metals Society of China 2011, 21, 202-207.

[7] Wei, Ch.K.; Davis, H.T.; Davis, E.A.; Gordon, J. Heat and mass transfer in waterladen sandstone: microwave heating. AICHE Journal 1985, 31(5), 842-848.

[8] Zielonka, P.; Gierlik, E.; Matejak, M.; Dolowy, K. The comparison of experimental and theoretical temperature distribution during microwave wood heating. Holtz als Roh- and Werkstoff 1997, 55, 395-398.

[9] Ratanadecho, P.; Aoki, K.; Akahoriu, M. Experimental and numerical study of microwave drying in unsaturated porous material. Int. Comm. Heat Mass Transfer 2001, 28(5), 605-616.

[10] Ratanadecho, P.; Aoki, K.; Akahoriu, M. Influence of irradiation time, particle sizes and initial moisture content during microwave drying of multi-layered capillary porous materials. J. Heat Transfer 2002, 124(2), 1-11.

[11] Zhang, D.; Mujumdar, A.S. Deformation and stress analysis of porous capillary bodies during intermittent volumetric thermal drying. Drying Technology 1992, 10(2), 421443.

[12] Rajagopal, K.R.; Tao, L. Modeling of the microwave drying process of aqueous dielectrics. Z. Angew. Math. Phys. 2002, 53, 923-948.

[13] Schiffmann, R.F. Microwave and dielectric drying. In A.S. Mujumdar (Ed.), Handbook of Industrial drying-1, New York: Marcel Dekker Inc. 1995, 345-372. 